\section{Tinnitus and Vertigo Symptoms: Precursors of Psychiatric Suffering}

Sir,

The symptoms of tinnitus and vertigo often originate due to the disturbances in the vestibulocochlear system. "Tinnitus is described as the perception of noise (ringing or humming in the ears), which is not from the external environment or as a result of auditory hallucinations associated with mental illness". 1 Vertigo or false impression of movement can be associated to a number of biological reasons linked with vestibulocochlear disorders. ${ }^{2}$

Recent studies have underpinned the concept that a variety of psychiatric issues like stress, anxiety, and depression may influence various disease activities among patients experiencing tinnitus and vertigo.3,4 The comorbidity of these symptoms along with psychiatric problems has been intermittently mentioned in the latest literature. Furthermore, it has been highlighted that the involvement of psychiatric features may delay recovery from tinnitus and balance disorders such as vertigo. ${ }^{3-5}$

The present study was conducted on a cross-sectional sample during the year 2018, spanning from the month of February to December at Hearts International Hospital, Rawalpindi along with Hearing and Balance Clinic, Rawalpindi. Two hundred and two patients were included in the study comprising of 60 females $(30 \%)$ and 142 males $(70 \%)$. Selected patients had vestibulocochlear problems for more than three months. Audiological assessments based on audiometry, tympanometry along with vestibular reflexes were performed to assess the retro-cochlear damage. Psychiatric assessments were performed by integrating tinnitus handicap inventory $(\mathrm{THI})$ and dizziness handicap inventory (DHI), depression, anxiety, stress scale (DASS) to gauge the severity of psychological symptoms along with physiological problems presented by patients. THI was significant, positively correlated with $\mathrm{DHI} \quad(r=0.38, p<0.001)$, depression $(r=0.63, p<0.001)$, anxiety $(r=0.59, p<0.05)$ and stress $(r=0.56, p<0.05)$. From the total sample, $27 \%$ patients were found to have tinnitus symptoms in both ears, around $64 \%$ patients had symptom of tinnitus in only one ear, and only $9 \%$ patients had symptoms of just vertigo. In the present study, around $80 \%$ patients had both concurrent vertigo and tinnitus symptoms. Moreover, it was noted in the patients experiencing vertigo and tinnitus symptoms that $17 \%$ patients were having normal hearing levels, $44 \%$ patients were having moderate hearing loss, around $35 \%$ patients had hearing loss of severe level, and only $5 \%$ patients had hearing loss of profound levels. Age ranged between 18 and 89 years (mean = $46.14 \pm=16.64$ ) of the selected sample reporting symptoms of tinnitus and vertigo from last three months.
Regarding the association of tinnitus and vertigo to psychiatric illness, it was observed that $54(26.7 \%)$ patients had very severe depression, $17(8.4 \%)$ had severe depression, $55(27.2 \%)$ had moderate depression, $25(12.4 \%)$ had mild level of depression, and $51(25.2 \%)$ were normal. Moreover, there were severe symptoms of anxiety 78 $(38.6 \%)$ and stress $149(73.8 \%)$ among patients. It was noted that female patients were more vulnerable to develop psychiatric symptoms than male patients. However, the prevalence of vertigo and tinnitus symptoms is more among male patients.

Current findings are in line with the past empirical literature highlighting that patients experiencing most provoking sensations of tinnitus and vertigo tend to have more prominent symptoms of anxiety, depression, and stress as compared to other bodily symptoms presented. ${ }^{3-4}$ Future researches can further examine the duration, gender differences and confounding reasons serving as sources of psychological problems among patients with vestibulocochlear disorder.

\section{CONFLICT OF INTEREST:}

Authors declared no conflict of interest.

\section{AUTHORS' CONTRIBUTION:}

AA: Wrote the whole manuscript and participated in literature review.

MA: Collected the patient data and images and participated in literature review.

BA: Provided audiological services and diagnosis of patients.

\section{REFERENCES}

1. Beukes E, Manchaiah V, Valien T, Baguley D, Allen P, Andersson G. Positive experiences related to living with tinnitus: A crosssectional survey. Clini Otolaryngol 2018; 43: 489-95.

2. Dieterich, Staab JP. Functional dizziness: From phobic postural vertigo and chronic subjective dizziness to persistent posturalperceptual dizziness. Curr Opin In Neurol 2017; 30:107-13.

3. Probst T, Dinkel A, Schmid-Mühlbauer G, Radziej K, Limburg K, Pieh $\mathrm{C}$, et al. Psychological distress longitudinally mediates the effect of vertigo symptoms on vertigo-related handicap. J Psychosom Res 2017; 93:62-8.

4. Bogo R, Farah A, Karlsson KK, Pedersen NL, Svartengren M, Skjönsberg A. Prevalence, incidence proportion, and heritability for tinnitus: A longitudinal twin study. Ear Hearing. 2017; 38:292-300.

5. Jastreboff $P$, Jastreboff $M$. Cognitive aspects of tinnitus treatments. J Hearing Sci 2017; 7:84.

Ammar Ahmed ${ }^{1}$, Muhammad Aqeell and Bashir Ahmed ${ }^{2}$

1 Department of Psychology, Foundation University, Islamabad, Pakistan

2 Department of E.N.T, Hearts International Hospital, Rawalpindi, Pakistan

Correspondence to: Dr. Bashir Ahmed, Department of E.N.T,

Hearts International Hospital, Rawalpindi, Pakistan

E-mail:ahmeddrbashir16@gmail.com

Received: April 17, 2019; Revised: June 20, 2019;

Accepted: June 26, 2019

$$
\text { ......... }
$$

